\title{
Development of 3D-Printed Heterogeneous Tumour Phantom for Quantitative Analysis in PET/CT Imaging
}

\author{
Ming Zhao Tang ${ }^{1}$, Hui Leng Choo ${ }^{1, *}$, Chai Hong Yeong ${ }^{2}$, and Yin How Wong ${ }^{2}$ \\ ${ }^{1}$ School of Computer Science and Engineering, Taylor's University, No. 1 Jalan Taylor's, 47500 \\ Subang Jaya, Malaysia \\ ${ }^{2}$ School of Medicine, Taylor's University, No. 1 Jalan Taylor's, 47500 Subang Jaya, Malaysia
}

\begin{abstract}
Tumours are heterogenous growths that consists of different types of cells with varying genetic expressions. The complex structure of the tumour makes cancer treatment difficult due to the heterogeneity of each of the cancer cells that react differently to radiation treatment. Therefore, effective treatment requires proper characterization of the tumour heterogeneity, which is difficult to be assessed by most of the current medical imaging technologies. The purpose of this study was to develop a 3D printed heterogenous tumour phantom with multiple compartments to simulate tumour heterogeneity and to assess the accuracy of tumour characterization using Positron Emission Tomography/Computed Tomography (PET/CT) imaging. The tumour phantom was designed and modelled using SolidWorks and then constructed using the fused deposition modelling (FDM) method of 3D printing with polylactide (PLA) filament as the material. Multiple layer of waterproof coating was applied and the phantom was carefully tested for any leakage prior to the study. Each of the compartments of the tumour phantom was filled with different activity of the radiopharmaceutical $\left({ }^{18} \mathrm{~F}\right.$ FDG). The phantom was then embedded in a water bath to simulate soft tissue and scanned under a PET/CT scanner using standard clinical protocol. The volume and radioactivity of each compartment were then measured using the clinical software as Standardized Uptake Values (SUVs). Results showed that PET/CT imaging able to delineate and quantify the radioactivity of each compartment within the tumour phantom, albeit some difficulties in detecting radioactivity below $100 \mu \mathrm{Ci}$. Further research is required to improve the design of the tumour phantom to allow for easier injection of the radioactive solution as well as altering the dimensions of the internal compartments to better characterize actual tumour parameters.
\end{abstract}

\section{Introduction}

Tumours are often heterogenous neoplasms, which are abnormal growths composed of different types of cells. Cancer treatment is made difficult due the complex structure of the various types of cells which have varying genetic expressions and signatures that mutate

\footnotetext{
*Corresponding author: huileng.choo@taylors.edu.my
} 
and react differently depending on numerous random intrinsic and extrinsic factors, also known as tumour heterogeneity. This makes development of an effective treatment difficult without properly understanding of the underlying heterogeneity of the tumour [1].

In malignant tumour cells, there exists different types of stem cells with characteristics that are highly phenotypic and diverse at both intertumoral (same tumour in different patients) and intratumor (distinct populations within the same specimen) levels. The reason for this heterogeneity is the dynamic ability the cells to adapt and evolve in a fluctuating environment. The stem cells do not conform to the instructions of the body and could be a result of both genetic and environmental factors that stimulate the mutations in the cell [2]. Some of these stem cells may be resistant to cancer treatments like chemotherapy. Thus, a single biopsy of only one small part of the cell may not be sufficient to capture the complete genomic landscape of the tumour. Treatments that are tailored to the biopsy sample might not be completely effective against the entire population of the tumour and might allow the surviving cells to proliferate and subsequently create a future resistant population of the tumour. This may also lead to recurrence of the cancer in the future. With proper analysis of the heterogeneity, the evolutionary drive of a cancer stem cell can be exploited to develop therapies that are more effective overall [3].

Biomedical imaging plays an essential role in the detection and management of cancer tumours. Early diagnosis and screening of potential tumours through advanced imaging techniques allows for improved treatment outcomes and reduced mortality. However, cancer cells mutate rapidly and adapt to survive in the human body, which can alter its reaction to radioactive treatments. Targeted imaging in tandem with biopsies can help to identify the genetic expressions of the tumour and metabolic changes, which will allow for the development of targeted therapies [4].

To improve upon the accuracy of imaging in cancer treatment, improvements in the image reconstruction protocols are needed as the current imaging technologies used cannot detect tumour cells that are smaller than a certain scale. Since repeated injections of radioactive material is harmful to the human body, a medical phantom is usually modelled and used as a substitute. Thus, the need for an accurate depiction of tumour heterogeneity that is suitable for imaging characterization [5].

The purpose of this research was to develop a heterogenous tumour phantom and assess the accuracy of Positron Emission Tomography-Computed Tomography (PET/CT) imaging on the characterization of tumour heterogeneity based on quantitative and qualitative analysis.

\section{Imaging Modalities in Tumour Characterisation}

Multiple types of imaging are used in all stages of cancer treatment to provide structural, morphological, metabolic and functional information about the tumour with the goals of reducing mortality of cancers. Most imaging techniques are based on the interaction of electromagnetic radiation on body tissues and fluids. These include nuclear imaging technique such as PET, as well as X-Ray technique such as CT. Other imaging modalities include Magnetic Resonance Imaging (MRI) and Ultrasound that do not utilize ionizing radiation [6]. Table 1 summarizes the advantages and disadvantages of each imaging modality.

PET imaging has been extensively used in cancer imaging due to its high sensitivity and specificity [8]. However, its limitation is the lack of anatomical identifiers which complicates treatment in tracking improvements with anatomical findings. This limitation can be compensated by combining the PET scanner with the CT to create a more detailed image that amalgamates the functional information from PET with the anatomical 
information from the CT [9]. Therefore, merging scanning modalities can improve cancer staging and diagnosis.

Table 1. Comparison of the advantages and the disadvantages between the different types of imaging

\begin{tabular}{|c|c|c|c|}
\hline \multicolumn{4}{|c|}{ modalities [7]. } \\
\hline & TECHNIQUE & ADVANTAGES & DISADVANTAGES \\
\hline PET & $\begin{array}{l}\text { Measures interaction } \\
\text { distance between } \\
\text { positrons and electrons }\end{array}$ & $\begin{array}{l}\text { High sensitivity and } \\
\text { resolution }\end{array}$ & $\begin{array}{c}\text { PET isotopes are } \\
\text { difficult and expensive } \\
\text { to synthesize }\end{array}$ \\
\hline Ultrasound & $\begin{array}{l}\text { Uses sound waves to } \\
\text { see inside soft tissue }\end{array}$ & $\begin{array}{l}\text { High resolution and } \\
\text { image contrast }\end{array}$ & $\begin{array}{l}\text { Low imaging depth with } \\
\text { many inaccessible parts } \\
\text { of the body }\end{array}$ \\
\hline CT & $\begin{array}{l}\text { Utilizes X-rays shining } \\
\text { through the body to } \\
\text { measure attenuation }\end{array}$ & $\begin{array}{l}\text { Capable of creating 3- } \\
\text { Dimensional images }\end{array}$ & $\begin{array}{l}\text { Requires contrast agents } \\
\text { of high atomic number } \\
\text { at high concentrations }\end{array}$ \\
\hline MRI & $\begin{array}{l}\text { Measures polarization } \\
\text { using a magnetic field }\end{array}$ & $\begin{array}{l}\text { Non-invasive and } \\
\text { relatively fast }\end{array}$ & $\begin{array}{l}\text { Difficulty with small } \\
\text { and flat lesions }\end{array}$ \\
\hline
\end{tabular}

\section{Standard Uptake Value}

PET/CT imaging is a noninvasive diagnostic tool that provides tomographic images that can be used to obtain quantitative parameter which in turn can be used to determine the metabolic activity of the targeted tissues. The imaging is made possible using a radiotracer known as Fluorine-18-Fluorodeoxyglucose $\left({ }^{18} \mathrm{~F}\right.$-FDG). It is a glucose analogue with a short half-life that emits positrons (subatomic particles that are emitted by radionuclides in patient tissue or organs) [10] and is taken up by living cells via the cell membrane glucose transporters. The distribution of the positron emitting radiotracers allow for threedimensional mapping of the tissue. Since higher glucose consumption is a characteristic of cancer cells, ${ }^{18}$ F-FDG PET has been proven to be a sensitive and effective tool for detection and treatment planning of various types of cancer as well as in monitoring the progression of the disease and responses to treatment [11].

The quantitative analysis on PET/CT images can be done using a dedicated software available on the PET/CT workstation. The analysis includes a clinical parameter known as the Standardized Uptake Value (SUV). The SUV is the ratio between the radiopharmaceutical concentration $(\mathrm{Bq} / \mathrm{mL})$ and the decay-corrected injected activity normalized by a given factor (mass of patient, body surface or lean body mass). Equation 1 shows all the parameters affecting the value of the standardized uptake according to the body weight.

$$
\mathrm{SUV}_{\text {bodyweight }}=\left(A_{c} \times W \times T_{1 / 2}\right) /-2 \times D \times \Delta T
$$

where $A_{c}$ is the activity concentration $(\mathrm{Bg} / \mathrm{ml}), W$ is the body weight $(\mathrm{g}), T_{1 / 2}$ is the physical half-life of the radionuclide (s), $D$ is the injected dose $(\mathrm{Bq})$ and $\Delta T$ is the delay between injection time and scan start time (s). 


\section{Design of The Tumour Model}

The heterogenous tumour phantom was designed using SolidWorks software. The size of the phantom was constrained to a diameter of $30 \mathrm{~mm}$, which is the general size of tumour before becoming large enough to pose a significantly increased health risk and becoming more difficult to treat [12]. The design of the tumour phantom was inspired by a normal spherical tumour which allows for the process of manufacturing to become significantly easier due to the lack of any difficult overhang shapes that are difficult for 3D printing. It also allows for easy alteration of the internal compartments in the 3D model using the software. As shown in Figure 1, the thickness of the walls between each compartment was set to $2 \mathrm{~mm}$ to provide support for the geometry and to prevent any fluid from leaking through the internal walls. Since the volume of each compartment must be known to allow for quantitative analysis of the imaging modalities, the internal geometry of the model was simplified to resemble a sphere.

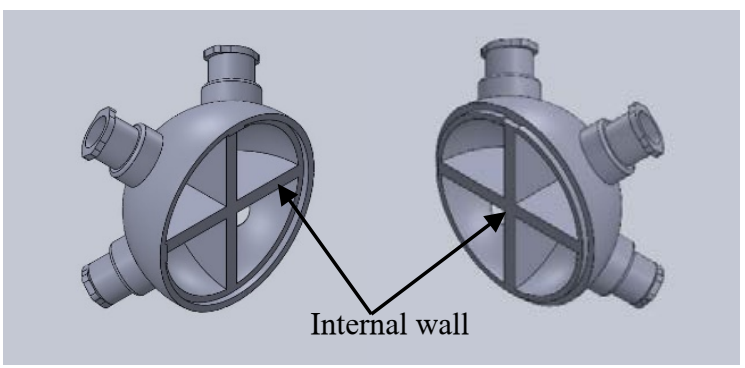

Fig. 1. Design of the heterogenous tumour phantom.

As shown in Figure 2, a compartment of the phantom is designed to be equal to a quadrant of a sphere. The internal volume of the compartments was determined using the tools provided in the SolidWorks software. Thus, the volume of each of the compartment was determined to be around $1790 \mathrm{~m}^{3}$. Each compartment can therefore hold a maximum of $1.79 \mathrm{ml}$ of the radioactive solution. This volume was used as the initial estimate for the radioactive solution to be injected into the phantom and to set a maximum threshold for the volume in the internal compartments.

To reduce the amount of supports from the $3 \mathrm{D}$ printing in the internal compartments, the model was split into two parts that could be reassembled later. The split parts were printed separately to prevent any errors in the printing and allow for more specific controls of the print settings. The inlet for injecting the radioactive solution was designed to be compatible with a Luer connector of a standard medical syringe. This connector allows the inlet to be universally applicable with most types of syringes. An additional plug was also printed to act as a seal for the inlets after the solution was injected.

The tumour phantom was printed using the fused deposition modelling (FDM) method of additive manufacturing with polylactide (PLA) filament. The material was selected due to its relatively low cost and environmental impact. The designed model was printed with a layer height of $0.1 \mathrm{~mm}$ and an extrusion width of $0.4 \mathrm{~mm}$. The infill density was set to the maximum of $100 \%$ to improve the water retention in each internal compartment and to reduce porosity of the phantom. The model was also printed from outside up to prevent any unnecessary supports that may affect the remaining volume of the compartments. The same model was printed three times to test the consistency of the print and to check for any errors in both the model design and the printing methods. 


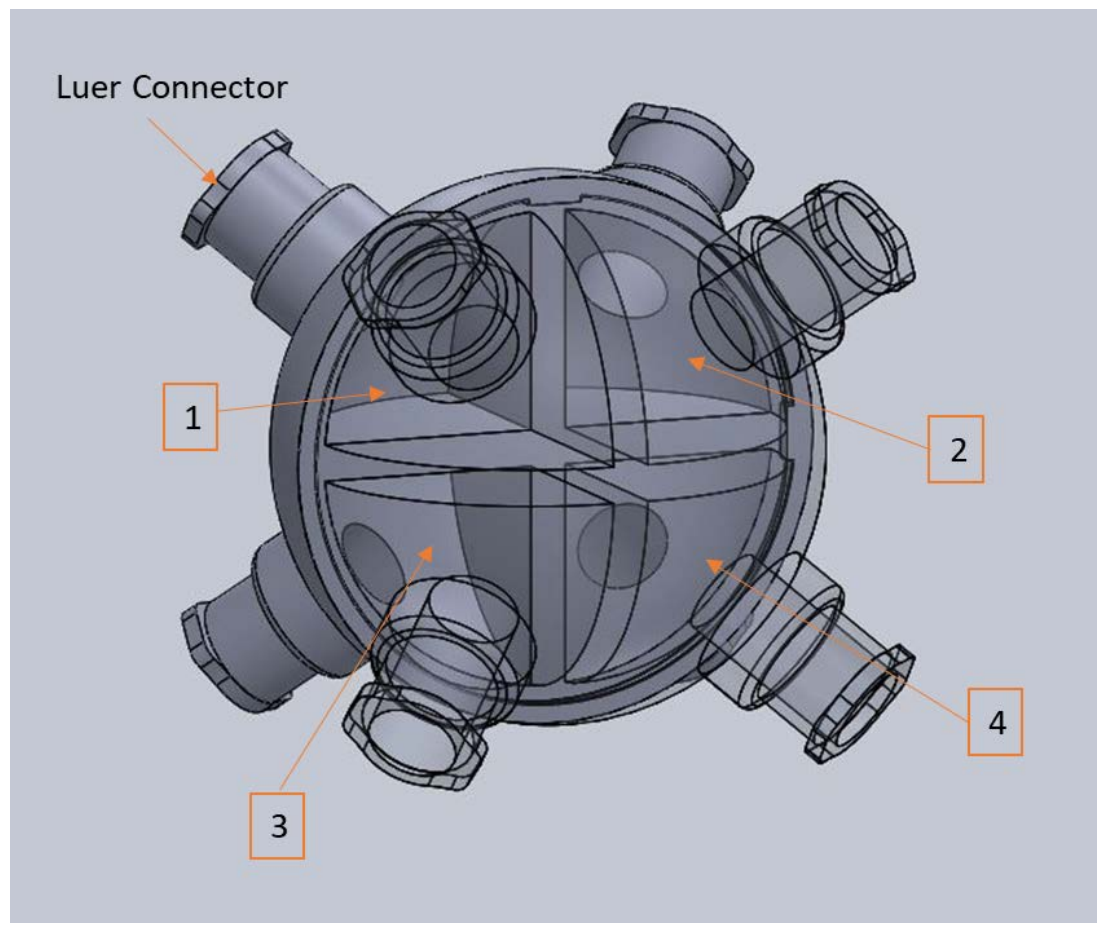

Fig. 2. Assembled model with visible internal compartments.

\section{Results and discussion}

Figure 3 shows the two-halves of the 3D printed imaging phantom. The two parts were sealed together using a silicon rubber-based sealant that was effective in creating a waterproof gasket in between the two parts. The printed model was observed to have slight imperfections in its geometry, especially at the edge of each inlet, as shown in Figure 4. The Luer design of the inlet head was damaged and was attributed to the limitations in the printer resolution. The printer used did not have the resolution needed to accurately replicate the model. However, since the internal compartments remain intact and the structural integrity of the model was not affected, the model could still be utilized for the purposes of data collection. The exterior discrepancies in the design will not affect the function of the phantom.

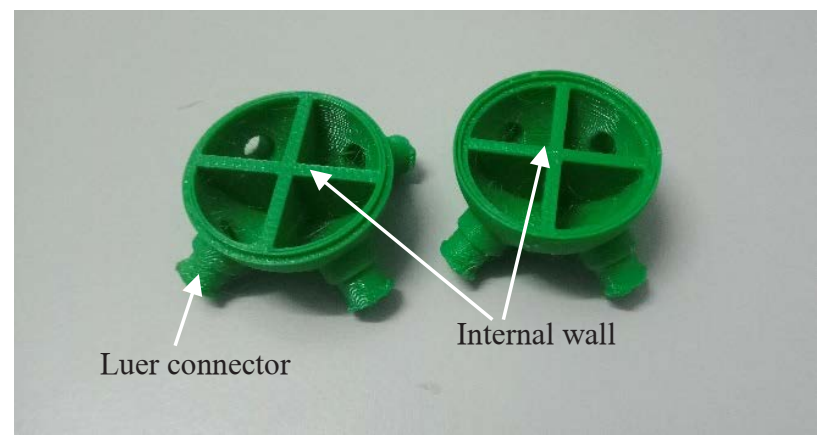

Fig. 3. Tow-halves of the 3D printed tumour phantom. 


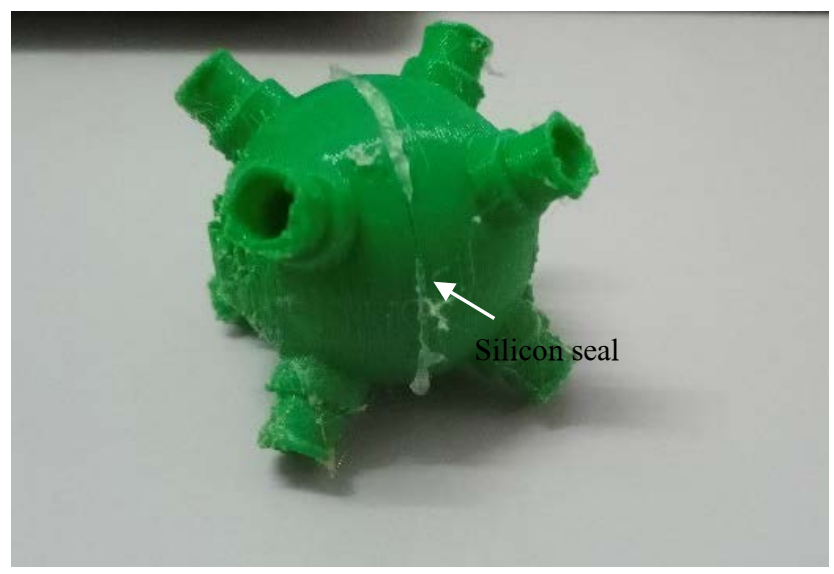

Fig. 4. The assembled model with silicon gasket.

The tumour phantom was tested for water solubility and absorption as well as its general porosity. The testing apparatus was shown in Figure 5. Red dye was diluted in water and injected into one of the compartments and then sealed with the printed plugs. The model was left to sit for some time to test its ability to retain the red dye. At the end of the testing period, the other three compartments were investigated for any red colored leaks to ensure that the model was fully functional in keeping fluid isolated into separate compartments. After confirming the viability of a single compartment, the other compartments were also tested similarly until the all the compartments were confirmed to be fully contained and functional.

The assembled tumour phantom was then taken to the study site for PET/CT scan. Different activities of the ${ }^{18} \mathrm{~F}-\mathrm{FDG}$, i.e. $1000 \mu \mathrm{Ci}, 500 \mu \mathrm{Ci}, 100 \mu \mathrm{Ci}$ and $50 \mu \mathrm{Ci}$ were injected into each of the compartments, respectively. The inlets were then sealed with waterproof medical tape and the phantom was submerged in a water bath to simulate surrounding soft tissue structures. The phantom was then placed under a PET/CT scanner (Biograph, Siemens, Germany) and scanned using standard clinical protocol. Figure 6 shows the PET/CT scanner used in the research.

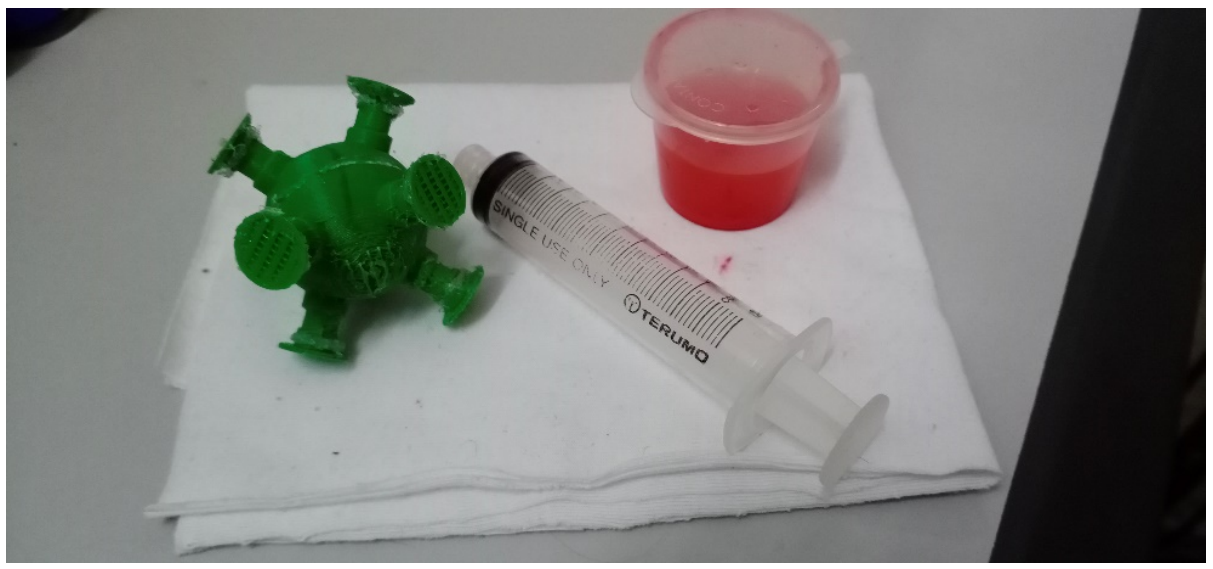

Fig. 5. Testing of the model with red dye. 


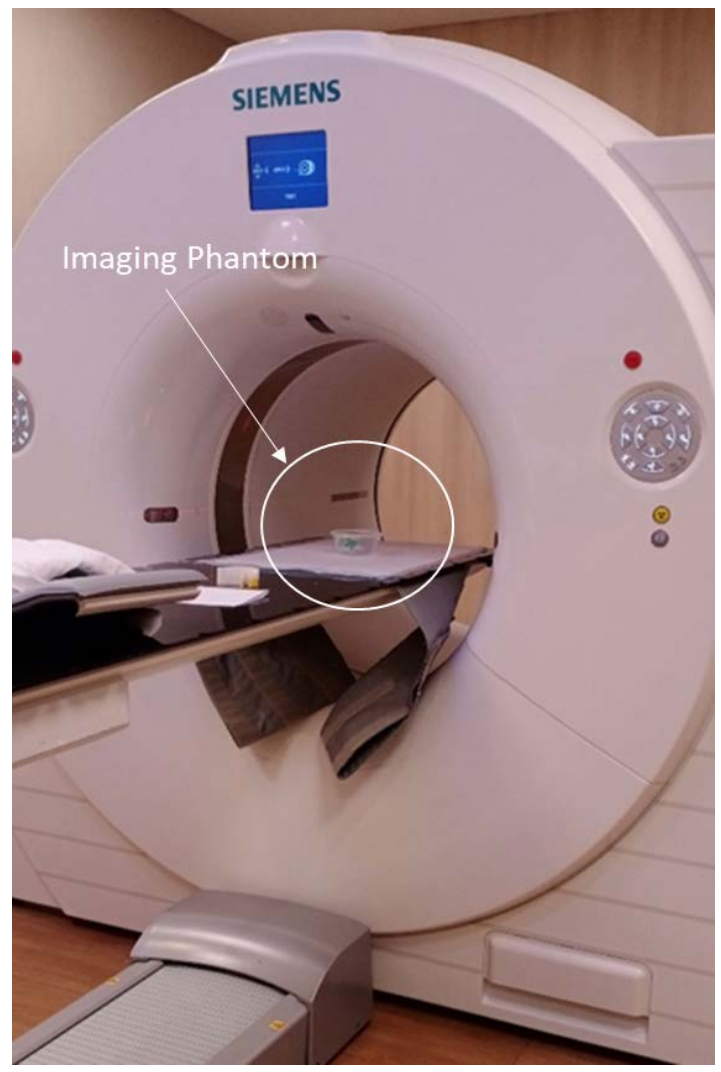

Fig. 6. Combined PET/CT imaging with the phantom in place for scanning.

After the scan was completed, the data obtained was reconstructed and analyzed using the proprietary imaging software in the hospital. Figure 7 shows the images created from the CT and PET scans. On the CT scan, a clear outline of the imaging phantom can be observed, with the internal compartments clearly visible as separate 3-dimensional sections. In contrast, the PET scan does not show a clearly defined shape, only the radiation activity can be observed.

To properly characterize the tumour heterogeneity, both types of imaging modalities must be combined to obtain a more accurate representation of the imaging phantom. Figure 8 shows the imaging reconstruction by combining both the PET image and the CT image to create a single image.

From the combined image, each separate compartment as well as its corresponding activity can be observed as different colored contours. The ${ }^{18} \mathrm{~F}-\mathrm{FDG}$ at the highest activity of $1000 \mu \mathrm{Ci}$ was observed to be in the compartment (a), with compartment (b) being the second highest activity at $500 \mu \mathrm{Ci}$. The remaining two compartments have no discernable differences and appear to have no visible contours, which shows the limits of the PET/CT scanner at detecting low amounts of activity through qualitative visual means.

After inserting the required parameters like total volume of fluid injected, the total injected dose and activity concentration, the imaging software can automatically determine the activity of a selected region of interest (ROI), in comparison to the surrounding fluid. SUV is the ratio of activity per unit volume for a specified ROI to the activity per unit volume of the entire body volume. This value is useful in the medical field for distinguishing abnormal tissues in the human body by comparing the levels of uptake. 

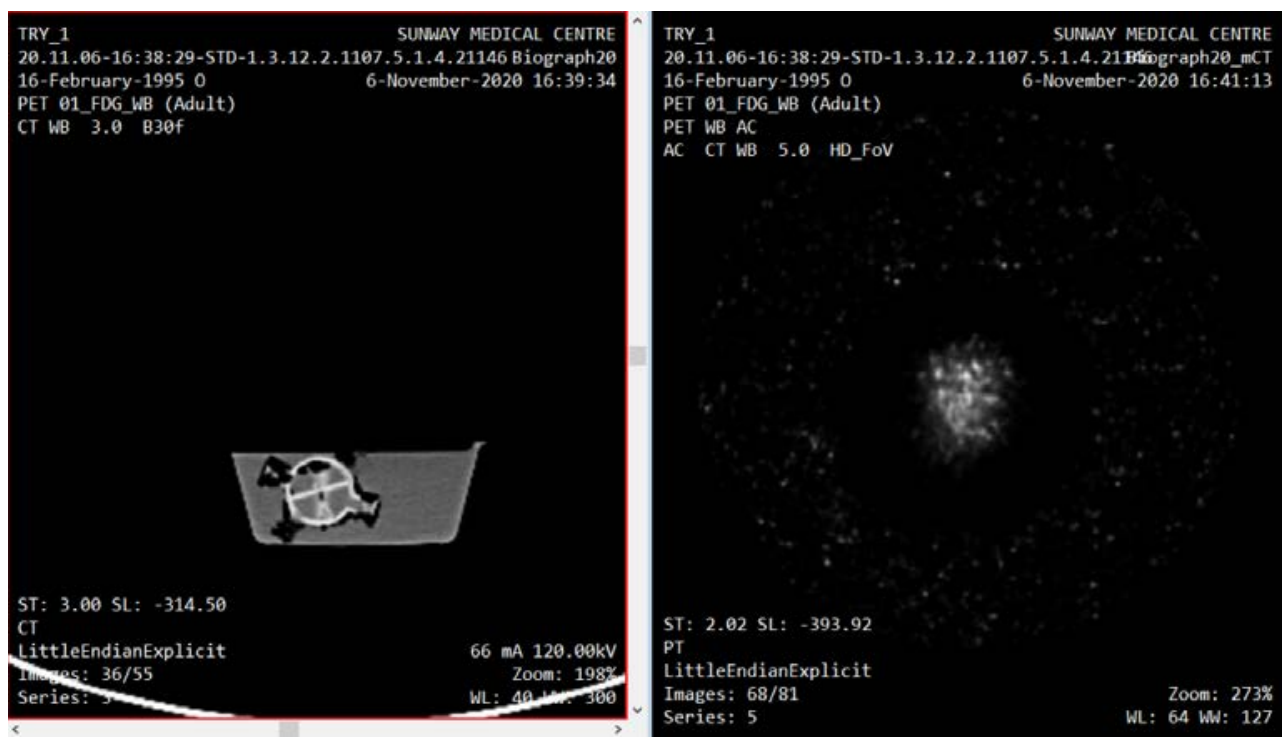

Fig. 7. CT Scanning (Left) and PET Scanning (Right) results.

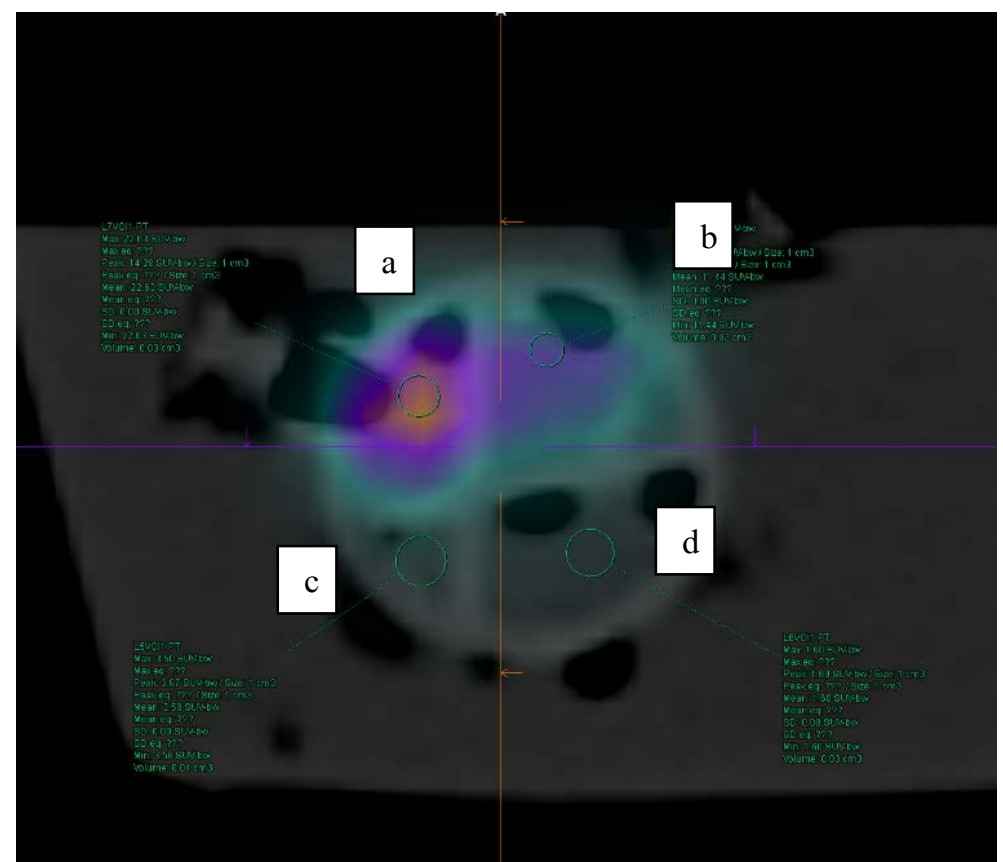

Fig. 8. Combined PET/CT Imaging showing compartments a, b, c and d.

Figure 9 arranges the compartments in ascending order of activity according to the calculated SUV values. Through this quantitative analysis, it was determined that compartment (a) presents a mean SUV of 22.6, (b) 11.4, (c) 3.5, and (d) 1.6. These values correspond to the activity of the radiotracer injected. This signifies that although the activity was not visible through qualitative means, it could still be detected by quantitative method. 


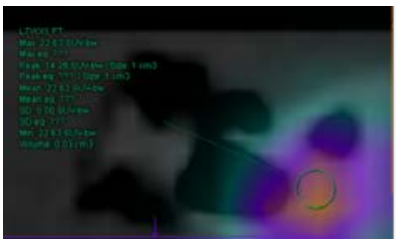

(a)

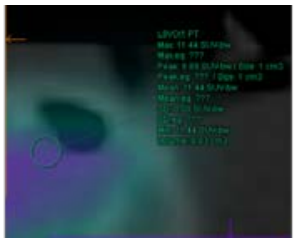

(b)

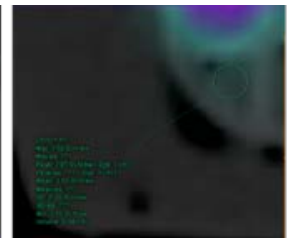

(c)

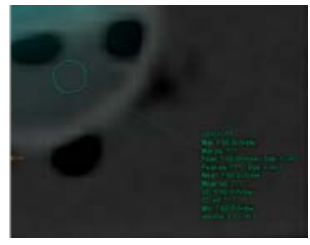

(d)

Fig. 9. Separated compartments in ascending order of activity (a) $1000 \mu \mathrm{Ci}$, (b) $500 \mu \mathrm{Ci}$, (c) $100 \mu \mathrm{Ci}$, and (d) $50 \mu \mathrm{Ci}$.

\section{Conclusions and Future Work}

A 3D printed heterogenous tumour phantom was successfully developed using 3D modeling techniques. The model was tested and proved to be fully functional and capable of retaining the radiotracer solution without leakage and was applicable for PET/CT imaging. The phantom was used to assess the accuracy of a PET/CT scanner in quantifying the radioactivity in each compartment by the mean of SUV. It was observed that the designed phantom was successful in simulating tumour heterogeneity in a tumour of $30 \mathrm{~mm}$ diameter and PET/CT scanning was able to delineate the compartments using quantitative SUV calculation. However, the PET/CT scanner was not able to detect activity below 100 $\mu \mathrm{Ci}$. Further research is required to improve the design of the model to allow for more sensitive and accurate imaging at a smaller scale.

Some recommendations for improvements in the future are to construct the model using higher quality tools and materials that allow for more accurate design details. An issue that arose during this experiment was the presence of air bubbles after injection of the fluid. The shape of the tumour phantom could also be altered to become more cylindrical which would allow greater volume to be inserted without worrying about air bubbles. The injection process of the radiotracer solution could also be streamlined by altering the design of the imaging phantom to have more safety precautions. Additionally, the internal volume of the model can also be modified to simulate actual tumour dimensions to produce more accurate imaging protocols that can be applied in clinical applications.

\section{References}

1. X. Sun, Q. Yu, Acta Pharmacol. Sin., 36, 1219 (2015)

2. R. Fisher, L. Pusztai, C. Swanton, Br. J. Cancer, 108, 479 (2013)

3. P. L. Bedard, A. R. Hansen, M. J. Ratain, L. L. Siu, Nature, 501, 355 (2013)

4. G. Lin, K. R. Keshari, J. M. Park, Contrast Media Mol. Imaging, 2017 (2017)

5. A. Valladares, T. Beyer, I. Rausch, Med. Phys., 47, 2023 (2020)

6. L. Fass, Mol. Oncol., 2, 115, (2008)

7. Z. Y. Chen, Y. X. Wang, Y. Lin, J. S. Zhang, F. Yang, Q. L. Zhou, Y. Y. Liao, Biomed Res. Int., 2014 (2014)

8. J. V Frangioni, J. Clin. Oncol., 26, 4012 (2008)

9. S. S. Anand, H. Singh, A. K. Dash, Med. J. Armed Forces India, 65, 353 (2009)

10. K. Kitamura, Oyo Buturi, 88, 47 (2019)

11. R. Boellaard et al., Eur. J. Nucl. Med. Mol. Imaging, 42, 328 (2015)

12. K. D. Yu, Y. Z. Jiang, S. Chen, Z. G. Cao, J. Wu, Z. Z. Shen, Z. M. Shao, Mayo Clin. Proc., 87, 1171 (2012) 